\title{
Conveying Emotions Through Device-Initiated Touch
}

\author{
Marc Teyssier, Gilles Bailly, Catherine Pelachaud and Eric Lecolinet.
}

\begin{abstract}
Humans have the ability to convey an array of emotions through complex and rich touch gestures. However, it is not clear how these touch gestures can be reproduced through interactive systems and devices in a remote mediated communication context. In this paper, we explore the design space of device-initiated touch for conveying emotions with an interactive system reproducing a collection of human touch characteristics. For this purpose, we control a robotic arm to touch the forearm of participants with different force, velocity and amplitude characteristics to simulate human touch. In view of adding touch as an emotional modality in human-machine interaction, we have conducted two studies. After designing the touch device, we explore touch in a context-free setup and then in a controlled context defined by textual scenarios and emotional facial expressions of a virtual agent. Our results suggest that certain combinations of touch characteristics are associated with the perception of different degrees of valence and of arousal. Moreover, in the case of non-congruent mixed signals (touch, facial expression, textual scenario) not conveying a priori the same emotion, the message conveyed by touch seems to prevail over the ones displayed by the visual and textual signals.
\end{abstract}

Index Terms-Touch, Social Touch, Social Touch Technologies, Robot Touch, Multimodal communication, Mediated communication, Embodied conversational agent, Facial expression

\section{INTRODUCTION}

The communication of emotions is multimodal. Many studies have focused on facial expressions [1], [2], vocal cues [3], [4], and body posture [5], [6]. Touch also plays an important role in conveying emotions [7], [8], [9]. Touch for communication is defined in Human-to-Human Interaction as an intentional contact to a relatively restricted location on the body surface of the receiver during a social interaction [10]. Touch stimuli can be characterized by several factors such as force, movement, velocity or repetition [7] and can convey various pro-social emotions. For instance, a soft stroke, a hit, or the placement of one's hand may respectively convey comfort, anger or calmness. Touch can reduce stress, encourage commitment or increase bonding between individuals [11]. Mediated touch can also increase realism and emotional communication in a variety of applications [12].

However, while touch plays an important role in humanto-human interaction, it has received limited attention from the Human-Machine Interaction community [11], [13]. Video games [14], mediated communication [7], care-related systems [15] (companionship, caring), or social robotics [15] often lack emotional communication through the touch channel. For instance, touch can increase immersion and sense of presence in co-located virtual environments [16], [17]. Similarly, an Embodied Conversational Agent ECA can use touch in addition to facial and body expression to communicate emotions [18]. While there have been some

- M. Teyssier and E. Lecolinet are with LTCI at Telecom Paris, Institut Polytechnique de Paris.

E-mail: contact@marcteyssier.com; eric.lecolinet@telecom-paristech.fr

- G. Bailly and C. Pelachaud are with CNRS at Sorbonne Université, in ISIR team.

E-Mail:

catherine.pelachaud@sorbonne-universite.fr

gilles.bailly@sorbonne-universite.fr,

Manuscript received June 17th, 2020; accepted July 7th, 2020. attempts in this direction, either for touching the user or "touching" the agent [13], most of such systems convey and perceive emotions through audio-visual channels [19], [20], [21]. Touch can also improve human-to-machine interaction in social robotics, for instance through the development of robot skin able to detect human touch [22].

Producing realistic touch remains a difficult challenge, and more knowledge is still needed about how the various touch parameters impact emotions [23]. Our aim is to understand how machine-produced touch can convey emotions and how it is perceived and interpreted by a human user. This first step is necessary to explore research questions such as when mediated touch should occur.

Similarly to [24], our approach consists of generating combinations of different touch parameters and asking participants to rate which emotions they perceive. After a pilot study, we conducted an experiment to understand which emotions can be attached to a set of touch parameters. Then, following [25]'s methodology, we conducted another experiment where we introduced a controlled context made of a textual scenario and a facial expression of an ECA. We rely on Russell's circumplex model of emotion representation through two dimensions, valence and arousal [26].

Our first challenge is to choose a device able to generate touch with sufficient naturalness. Considering the limitations of current technology, we choose to use a robotic arm augmented with an artificial hand that touches the user's forearm (Figure 2). We first motivate the choice of the device as well as how the device is going to touch (selection of touch characteristics). We conduct a pilot study to validate the device and select the most relevant touch factors.

We then conduct two different dependent studies. Study 1 explores how the combination of touch factors is perceived in terms of arousal and valence, without any information regarding the interaction context; that is participants receive 
just the touch factors as stimuli. Study 2 allows understanding how interaction context (here given by short textual scenario and virtual agent's facial expression) impacts on the perception of arousal and valence.

Our paper is structured as follows. After introducing the related work, we present our general approach. We then describe the choice of factors and apparatus. We then present the experimental design, and tasks conducted in the three studies. Their results are presented and discussed. Finally we conclude the paper by discussing the main findings of our work and present future directions.

\section{Related Work}

During Human-to-Human communication, people use touch to establish bonds or trust between people, to convey affects, etc. [27]. Touch gestures that communicate emotions are rich and complex [7], [8], [9], [28]. For instance, the emotion Anger can be perceived through a short and localized gesture (hit) whereas Comfort can be perceived with a slow and protracted gesture with back-and-forth movements (stroke) [7]. In this section, we report on physical characteristics that compose human touch, and present haptic technologies and systems that convey emotions through touch.

\subsection{Touch for emotional communication between hu- mans}

During Human-Human interaction, emotions can be conveyed by touch only [8] through the variation of spatiotemporal characteristics of touch such as duration, location, intensity, velocity, abruptness or temperature [7], [9], [28].

Touch can be characterized by several spatio-temporal characteristics. First, Spatial characteristics include the $0^{-}$ cation where the touch is applied on the body [29]. The forearm, the shoulder and the back are the locations that are generally the most socially and culturally accepted [8] and are suited for social and intimate relationships [30]. The touching area or surface of contact depends on the kind of touch gesture (e.g. a hug vs. a touch with the fingertip) [31]. Next, Temporal characteristics characterize the dynamics of touch movements. They include the duration of a touch [32], the velocity of a touch movement [9], [28], and repetition and spatio-temporal patterns, which can either involve a succession of strokes (e.g. pats) or back-and-forth movements on the skin [33], [34]. Finally, other characteristics impact the perception of emotions through touch such as force intensity through variation of pressure [35], Texture [28] or the Temperature of the end-effector [36], [37].

These individual characteristics have an impact on the perception of emotions. Low velocities are more associated with pleasant emotions. Negative emotions tend to involve a short duration, and high velocity, high pressure [7]. The impact of temperature on emotions has to be clarified with studies finding that cooling has positive valence [38] or the opposite [37]. Regarding the texture of the end-effector, smooth, soft materials received higher pleasantness ratings than rough, coarse materials [28].

In summary, the design space of generated touch is enormous as it not only involves all the characteristics listed above but also the combination of these characteristics and their parameters. To study machine-produced touch in a controlled experiment it is thus necessary to first reduce the size of the design space. It is then possible to precisely study the impact of a subset of touch parameters on emotions. The choice of the parameters should been circumscribed by the results reported on human touch by previous studies [7], [8], but also by the constraints of current technology generating touch.

\subsection{Generating Touch: Tactile and Kinesthetic feed- back}

For tactile feedback vibration motors remain the primary tactile actuators that are used to convey tactile feedback and study emotional touch [33], [39], [40], [41], probably because they are inexpensive, easy to use, and already embedded in many devices (e.g. smartphones). However, this technology was not initially designed for emotional communication and it can only produce a subset of the sensations that humans are able to perceive. The actuators have to be in direct contact on the skin at all times and rely on patterns to perform dynamic touch. Moreover, these types of actuators seem to primarily impact the perceived level of arousal [38]. However, they can be combined with other modalities to expand their emotional range [42].

Other technologies include thermal feedback [43] or shape-changing interfaces with pneumatic actuation [44], [45], [46]. The projects relying on these technologies generally focus on interaction techniques (e.g. notification, guidance) rather than on their capacity to convey emotions.

Kinesthetic feedback usually involves larger devices. Force feedback actuators [47] have been investigated to convey emotions, were rated as more natural, and showed a greater emotional interdependence with a stronger sense of copresence than vibrotactile touch [17]. They are also more adapted to perform large-scale haptic feedback [48] as they can go around the user and produce contact at various body locations. Robotic devices have been used to provide passive haptic feedback [49], [50], [51]. Using these devices in a virtual reality environment is relevant as the physical robots are hidden from the user's sight when wearing an HMD. In this example, the user has to touch the robot making it unsuitable for the device to communicate emotions. On the other hand, we are interested in scenarios where the robot touches the user.

Zoomorphic robotic creatures are often used to convey emotions through movement and touch [52], [53]. They simulate social interaction and often use a hug as a means to convey positive emotions, reduce stress and anxiety [54], [55]. Some studies suggest that a robot-initiated touch could be perceived as human contact [56], and others report that people prefer touching robots than being touched [57]. More recently, robotic swarms were used to perform social touch [58]. Our approach draws inspiration from these studies and also focuses on robot-initiated touch, where the device performs protracted and dynamic contact on the user's skin.

\subsection{Combining Visual and Touch Cues}

Emotions can also be conveyed through facial cues. Ravaja et al. [59] report on a study of affective modulated touch 
perception through facial expression. Participants rated the same touch as more intense when preceded by an angry, happy and sad facial expression [59]. This phenomenon was also observed when touch is produced concomitantly with the display of facial expressions of virtual characters [19], [60], [61]. Although these studies report some differences in emotional touch perception when visual stimuli is also presented, it is not clear how it impacts the arousal and valence rating. This can be influenced by the device that performs touch, for instance the type of device and actuation method or the location of the touch stimuli on the body. As there is no standard protocol to display the facial expression, the duration of presentation of facial stimuli or the context can also influence the rating.

This paper primarily focuses on the perception of emotions through touch. However, because emotions are often conveyed through multiple channels, we also explore the impact of the context through visual and textual cues.

\subsection{Assessment of Touch to Convey Emotions}

Scholars have proposed several representations of emotions such as labels [1], dimensions [26], [62], and appraisal [2]. Emotions are characterized by specific behavioral patterns, physiological responses and functions. In the emotional touch domain, Russell's circumplex model of emotions [26] is the most frequently used. It represents emotions along a two dimensional space where the $x$ axis refers to valence (emotional pleasantness) and the $y$ axis refers to arousal (activation/excitation). In our study, we rely on this model and refer to it as the arousal/valence space. Moreover, in this model, every point on the arousal/valence space can be linked to an emotion. For instance, the emotion Anger is represented in the top-left quadrant (high arousal, low valence) while the emotion Happiness is represented in the top-right quadrant.

\section{General approach}

In this paper, we study how humans perceive and interpret machine-produced touch in terms of arousal and valence. Our overall approach consists of transposing emotional perceptual experiments conducted in human-to-human interaction studies to machine-to-human interaction. This approach is frequently used in ECA literature, for instance to understand and reproduce the effect of smiling during conversation [25]. We built on top of this related work to develop our approach described below:

Selecting Touch Characteristics and Device. We first selected the characteristics that will be explored in controlled experiments. Generating touch requires considering its various characteristics and their associated parameters. This raises the challenge of choosing an appropriate device. These two aspects need to be considered together as current technology limits the set of factors that can be implemented, thus investigated. For instance, controlling force, temperature, and skin moisture simultaneously might be technically difficult. These choices (set of touch parameters and device) were performed iteratively, by considering previous literature on human-to-human interaction and the characteristics of touch-generating devices (see Section 2). Section 4.1 describes the set of touch parameters we chose (Amplitude, Velocity, ForCe and TyPe) and Section 4.2 presents the device that we selected (a robotic arm augmented with an artificial hand).

Pilot study: Evaluation of touch factors. The role of the pilot study presented in Section 5 was to introduce the experimental setup and to identify the most promising characteristics and their values for conveying different degrees of arousal and valence. This allowed us to select touch parameters with the most significant effects in the perception of emotions. We retained three characteristics (AMPlitude, Velocity and FORCE).

Study 1: Perceptive Study on Context-Free Generated Touches. The first experiment presented in Section 6 investigated how humans perceive eight machine-generated touches (the combination of the three characteristics executed with two small and large values). It allowed us to select certain touch parameters that tend to have distinct effects in the perception of emotions in terms of valence and arousal. This study was done context-free, meaning that no information other than the touch stimuli was given to the participants. We applied a methodology similar to [25], often used in perceptual studies. The notion of context in our approach refers to the context of social interaction.

Study 2: Perceptive Study on Generated Touches with Context Cues. Finally, in Section 7 we describe an experiment that investigates how context modulates the perception of machine-generated touch [63]. Context is defined here by textual scenarios and facial expressions of a virtual agent. The scenarios have been designed following the methodology proposed by Scherer et al. [2]. They correspond to one (non ambiguous) emotional situation and have been used in other studies [25], [64], [65]. The visual cues correspond to the facial expressions of a virtual character of the emotions associated to the scenarios. With this experiment we aim to understand how context impacts on the perception of the touch stimuli in terms of arousal and valence. This study brings us closer to examining the perception of emotions through multimodal signals, namely facial expressions, touch and text that provides information of the events that trigger the agent's multimodal response.

In the following sections, we provide further in-depth details on the four aforementioned parts.

\section{Selecting touch Factors and DeVICE}

In this section, we explain the choice of touch parameters and of the device. While reported sequentially in this section, these choices followed an iterative process. It is important to point out that our investigation was not technologically driven and did not focus on a specific device (e.g. smartphone [42]), which is common in the HCI community. In contrast, we started by considering human factors.

\subsection{Selecting touch characteristics}

We broke down touch movements present in the humanto-human literature [7], [8], [9], [28] by considering them 
as a composition of simple characteristics. Given the numerous characteristics, we retained four of them: AMPLITUDE, VELOCITY, FORCE and TYPE. We chose these four characteristics because they allow us to generate a wide range of touch gestures including Hitting, Stroking, Patting, Contact or Tapping. These gestures are often associated with a wide range of emotions [7], [8], [9], [28]. We excluded some gestures for the reasons explained below, but that are worth exploring in future studies. For instance, we excluded gestures that require holding on to someone (e.g. Grabbing, Lifting the arm) or protracted touching on the back (Hugging), as they would require complex interweaving between the machine and the participant. We also did not consider touch characteristics such as temperature or skin moisture. These characteristics do not seem to have a clear effect on the perception of emotions [37], [38], [42]. Moreover they are not easily reproduced on current devices. Finally, we decided to consider gestures performed only on the forearm. This location is generally suitable in most cultures [30], [66] and commonly used in human-device studies [33], [60].

\subsection{Selecting the device}

Our primary motivation when choosing a device was its ability to produce human-like touch, i.e. its capacity to generate touches similar to those generated by humans. We thus quickly excluded vibration motors and focused on technologies providing kinesthetic feedback as well as tactile feedback close to those produced by the palm and fingers of the human hand. Inspired by robot-initiated touch in human-robot interaction [23], [48], [58], [67], we decided to use a robotic arm augmented with an artificial hand.

Regarding the robotic arm, we chose to use a 7-degreeof-freedom KUKA LWR4+ compliant robotic arm (Figure 6, left), originally designed for safety with collaborative robotic applications in mind. This robot enables accurate motion and ensures the security of the participant. The workspace of the robot is around $1.5 \mathrm{~m}^{3}$, the amplitude can be precisely controlled, and the end-effector can be reliably positioned in a $3 \mathrm{D}$ space $( \pm 0.05 \mathrm{~mm})$. The velocity of the end-effector can be accurately varied from $2 \mathrm{~cm} / \mathrm{s}$ to $40 \mathrm{~cm} / \mathrm{s}$. We used a Microsoft Kinect V2 to track the anatomy of the user's arm, to ensure the robot's hand follows the user's arm morphology, and to adapt the force intensity along her arm. The force is measured with a dedicated apparatus connected to the end-effector of the robot (ATI F/T Sensor Mini45). Masses and spring constants of the lower arm skin are similar between individuals and are around $40 \mathrm{~N} / \mathrm{mm}$ [68]. Hence the touch was applied along the surface of the human arm with an offset of the position of the end-effector relative to the target force $( \pm 0.1 \mathrm{~N})$.

Regarding the artificial hand, we used a silicon human hand attached to the robot (inspired by prosthetic hands bionics).

\subsection{Selecting touch parameters}

Once the choice of device was finalized, we selected the parameters of each different touch characteristic. In the following paragraphs, we use the term velocity $(V)$ as the velocity projected on the axis of the forearm. We distinguish between dynamic movements $(V \neq 0)$ and static movements $(V=0)$. Dynamic movements, such as a pat on the participant's arm, are characterized by the change of location of the end-effector (here, the hand attached to the robotic device) over time. In contrast, a static movement is performed at the same location (but can also have different duration and repetition characteristics) [7]. The selected touch parameters are presented in Figure 1 and are all in range with the mechanical constraints of the robotic arm.

\subsubsection{Dynamic touch movement}

We monitor four characteristics in the dynamic condition:

AMPLitudE (labeled A) represents the length of the movement performed on the arm. We consider two amplitudes, $5 \mathrm{~cm}$ (labeled $A-)$ and $20 \mathrm{~cm}(A+)$, which are commonly used in the human-touch literature [9].

VELOCITY indicates how fast the gesture is performed. We consider two velocities, $16 \mathrm{~cm} / \mathrm{s}(V+)$ and $3.8 \mathrm{~cm} / \mathrm{s}(V-$ ). Their difference $(\operatorname{diff}=12.2 \mathrm{~cm} / \mathrm{s})$ is similar to the values used in previous human touch experiments $(\mathrm{diff}=15 \mathrm{~cm} / \mathrm{s})$, and seems to be sufficient to signal different emotions [9].

The DURATION of a movement can be derived from its amplitude and velocity. It varies between short (0.3s), medium (1.3s), and long (5.2s).

With FORCE, we consider two levels, low $<0.3 \mathrm{~N}(F-)$ and strong $>1.2 \mathrm{~N}(F+)$. The FORCE range varies slightly from participants due to their forearm morphology, but still ensures a perceptible difference between the two levels and remains small enough not to hurt the participant's arm [35].

For TYPE, we consider three repetitions of movements: Simple (T0), Pat (Tp), and Stroke (Ts) [7], [69]. A Simple gesture is a one-directional movement from one position to the another on the forearm. Pat is a 4-time repeated gesture and Stroke a 2-time back-and-forth gesture.

\subsubsection{Static touch movement}

When considering static touch movements, AMPLITUDE and VELOCITY are null. We thus only consider FORCE and DURATION parameters in this case. The values for DURATION (Labelled $D$ ) are $0.3 \mathrm{~s}\left(D^{-}\right)$and $1.3 \mathrm{~s}(D+)$, in order to be comparable in static and dynamic conditions. Regarding the TYPE of the gesture, we consider Simple (TO) and 4Tap (Tp). 4-Tap is an adaptation of Pat without movement

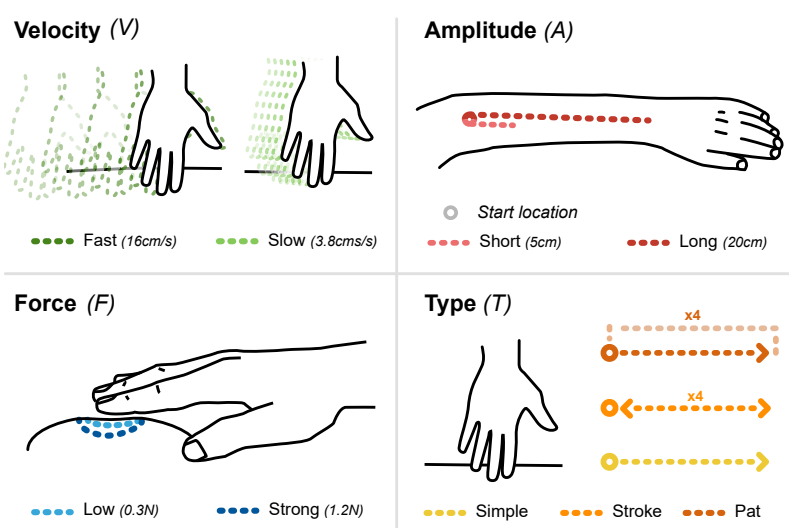

Fig. 1. Touch characteristics used in the studies, and their parameters. Characteristics include Velocity, Amplitude, Force and gesture Type. 
on the arm. The Stroke gesture is removed because there is no displacement.

\subsection{Generated touches}

In total, there are 24 dynamic generated touches (2 AMPLITUDE $\times 2$ VELOCITY $\times 2$ FORCE $\times 2$ TYPE) and 8 static generated touches (2 TYPE $x 2$ DURATION $\times 2$ FORCE). The generated touches are labeled with the acronyms of the touch parameters. For instance, $V-A+F+T s$ corresponds to a generated touch with the smallest VeLOCITY $(3.8 \mathrm{~cm} / \mathrm{s})$, the largest AMPLITUDE $(20 \mathrm{~cm})$, the highest FORCE $(>1.2 \mathrm{~N})$ and a Stroke TYPE.

In conclusion, we drastically reduced the size of the design space of touch by considering four characteristic and two different values per characteristic. The device is able to reproduce all of these parameters. We conducted a pilot study to understand the pertinence of the 32 generated touches in conveying different values of arousal and valence.

\section{Pilot study: Evaluating touch factors}

The objective of this pilot study is to compare the 32 previously defined machine-generated touches in order to identify the most promising characteristics and their values for conveying different degrees of arousal and valence. No contextual cues are used in this experiment.

\subsection{Apparatus}

The setup is illustrated in Figure 2. Participants are seated at a table, in front of a computer screen, wearing earphones that produce white noise as well as noise-cancellation headphones to hide the sound of the robotic arm. The left arm of the participant is laid on the table with the palm oriented downwards so that the stimuli are applied on the back of the forearm.

The experimental software for controlling the robotic arm is implemented within Unity, the tracking device that allows us to place the starting point of the touch movement near the elbow (Figure 1, Amplitude) and to follow the anatomy of the user's forearm in order to apply a constant force. Several layers of security are implemented to ensure the participant's safety, including robust user tracking, precise inverse kinematics, as well as the definition of a limited and well-identified interaction zone.

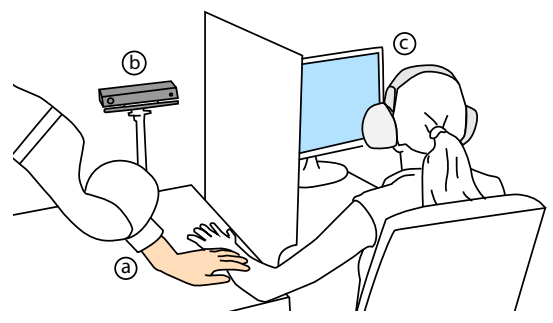

Fig. 2. Setup of the experiment. a) The robotic arm is hidden behind an opaque screen and touches the user's forearm. b) A Kinect sensor tracks the user's forearm to follow its arm anatomy. c) Participant wears both earphones emitting white noise and a noise-cancellation headphone to hide the sound of the robotic arm.

\subsection{Experimental design}

For comparison purposes, the procedure is similar to other studies investigating human-to-human touch [7], [9] and assessing emotion perception [70]. Sixteen volunteers (7 F) who are all right-handed students and staff members from the same academic institution, with a mean age of 26.4 $(\sigma=1,9)$ took part of the study. They are provided with a description of the task, "This study will assess which emotional content you think is being represented by each stimulus." Participants saw the robotic device prior to the study. We informed the participants that the stimulus is performed by a robot touching their arm and that they have to assess how they perceived the levels of arousal and of valence are conveyed by this touch stimulus. Once participants are ready, they interact (with a mouse in their right hand) with the experimental interface to start the study. The experimental device then executes the stimulus, i.e a generated touch composed by a combination of parameters. Once the generated touch is executed, the participants fill in a questionnaire displayed on the screen about the touch they perceived and their emotional experience [59]. To reduce cultural confound factor, participants in the studies are all French.

We measure the perceived emotions using the dimensional representations of emotions (Valence-Arousal emotional space [26]). The first part of the questionnaire assesses the arousal/valence rating: "Was the emotion conveyed by touch pleasant?" and "intense?" on a 7-point Likert scale (e.g. 1: "not at all pleasant", 7: "very pleasant"). We explain to the participants that "intense refers to physiological arousal or excitement".

At the end of the experiment, participants are given another questionnaire (on a 7-point Likert-scale) about their overall experience and their perception of the robotic arm and their sensibility to touch. They are asked to indicate how much they agree with the following statements: You touch the other speaker during a conversation; You enjoy being touched during a conversation; This device is adapted to perform touch; The touch stimuli presented are efficient to convey emotions; The touch stimuli were human-like; It was difficult to associate touch stimuli with an emotion; You accept to be touched by a robotic arm.

The pilot-study follows a within-participant design, with the order of presentations of each condition counterbalanced between participants. Each stimulus is repeated three times during the experiment. We obtain a total of 96 stimuli ratings per participant $(8$ static +24 dynamic movements) $\times 3$ repetitions. Participants are given a break of 10 seconds between each trial and a longer break after 12 stimuli to let their arm rest. Overall, the study lasts between one hour to 90 minutes.

\subsection{Results}

We remove $3.0 \%$ (35/1152) outliers based on arousal and valence ratings using the Wilks' method [71] on the dataset containing the dynamic movements and $1.3 \%(5 / 384)$ on the dataset with static movements. The main findings remain the same when outliers are included in the analysis. Moreover, our results suggest that fatigue did not impact Arousal/Valence (A/V) ratings; both MANOVA and 

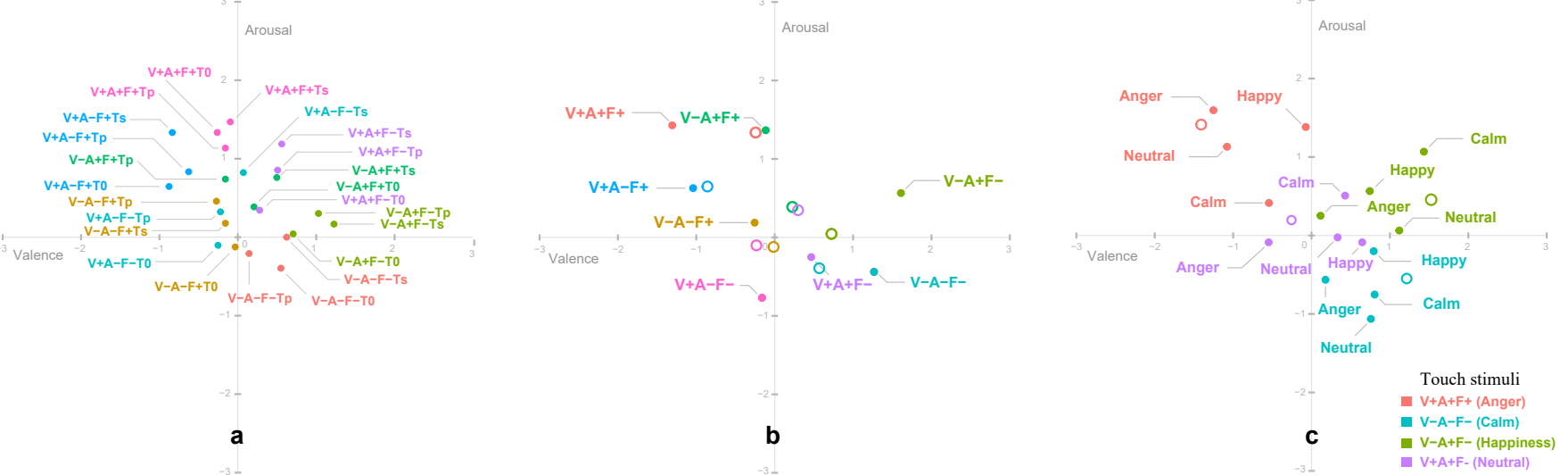

Fig. 3. Results of arousal/valence distribution for all the perceptual studies. a) Pilot study :) with all generated touches. b) Study 1: Context-free) with the relevant three characteristics (Velocity, Amplitude, Force). Larger circles are the stimuli from the pilot study. c) Study 2: With context effect of context cues on generated touch stimuli. The context cues are labeled on each point, the color corresponds to a touch stimulus. Larger circles are the stimuli from the context-free study.

ANOVAs reveal no effect of presentation order of stimuli $(1,2,3)$ on Valence and Arousal.

To determine effects on individual measures (effect sizes are shown as $\eta_{p}^{2}$ ), we analyze the ratings using a two-way repeated-measure MANOVA with valence and arousal as combined dependent variables. For the dynamic movements, MANOVA finds significant main effects for the characteristics VELOCITY $\left(F_{(2,1127)}=100.56, \mathrm{p}<0.001\right.$; Wilks $\left.\Lambda=0.86, \eta_{p}^{2}=0.13\right)$, FORCE $\left(F_{(2,1127)}=54.78\right.$, $\left.\mathrm{p}<0.001 ; W_{i l k s} \Lambda=0.86, \eta_{p}^{2}=0.12\right), \quad$ AMPLITUDE $\left(F_{(2,1127)}=29.67, \mathrm{p}<0.001 ;\right.$ Wilks $\left.^{\prime} \Lambda=0.95, \eta_{p}^{2}=0.05\right)$, but not effect on TYPE.

For static movements, MANOVA reveals a significant effect on FORCE $\left(F_{(2,375)}=16.78, \mathrm{p}<0.007 ;\right.$ Wilks $^{\prime} \Lambda=0.92$, $\left.\eta_{p}^{2}=0.08\right)$ and a small effect on touch TYPE $\left(F_{(2,375)}=7.12\right.$, $\left.\mathrm{p}<0.001 ; w^{\prime} \Lambda=0.96, \eta_{p}^{2}=0.04\right)$ and no effect on DURATION nor interaction effects. The distribution of arousal/valence rating for each stimulus (static and dynamic touch) is presented in Figure 3, $a$.

\subsection{Discussion}

Our results suggest clear effects of dynamic touch (involving the parameters Velocity, ForCe, AMPLitude). While we anticipated a strong effect of the TYPE characteristic on the perceived arousal and valence when performing dynamic touch, this was not the case. This hypothesis was influenced by previous research that has reported on the impact of repetitive sequences of touch to convey different meanings [69]. Our study does not completely reproduce results from a number of previous studies [7], especially in the static condition, but, these studies also considered some touches (e.g. grab, hug) that our device could not produce. Considering the effect of static touch on arousal and valence (involving the characteristic DURATION, FORCE, TYPE), the impact is less clear for participants in our experiment.

Considering the result of this pilot study, and the fact that some results were not significant, we decided not to consider the TYPE characteristic and static touches in our next studies. We therefore focus on eight machine-generated touches which are a combination of the parameters of AMPLITUDE, VELOCITY and FORCE. These combinations still enable us to perform touch gestures such as Hit, simple
Stroke or Pat, but exclude prolonged bi-directional strokes or multiple taps.

\section{Study 1: Investigating Context-Free GeN- ERATED TOUCHES ON EMOTION PERCEPTION}

The objective of the experiment described in this section is to confirm the findings of the pilot study and to precisely understand the impact of the characteristics and parameters on emotion perception in a context-free situation.

\subsection{Experimental Design}

The apparatus and the experimental design of this study are similar to the pilot study. From the result of this first study, we did not consider static generated touches and the TYPE characteristic. This experiment consists of $2^{3}=8$ different stimuli corresponding to the combination of two values (low / high) for each of the three characteristic (VELOCITY, AMPLiTUdE, FORCE). Each stimulus is repeated twice. The experiment lasts for about 15 minutes. Thirty-two volunteers $(14 \mathrm{~F}, 18 \mathrm{M})$ of the French culture participate in the study, with a mean age of $26.5(\sigma=6)$.

\subsection{Results}

We map the average arousal/valence results obtained for each individual stimulus onto the circumplex model [72]. To fit the two dimensions of the model, we convert the 7-point Likert-scale ratings to -3 to +3 scales; each pair of arousal and valence values is taken as a coordinate in the 2D space. The values for the majority of the stimuli (Figure $3, b)$ lays within the 'high arousal, low valence' quadrant (top-left), associated with emotional states such as anger or frustration. There are a smaller number of points in the 'low arousal, high valence' quadrant (bottom-left: satisfaction, calm) and one point found within the 'low arousal, low valence' quadrant (bottom-left: depression, sadness, tired) and the 'high arousal, high valence' quadrant (top-right), associated with emotions such as excitement, happiness, and amusement. 


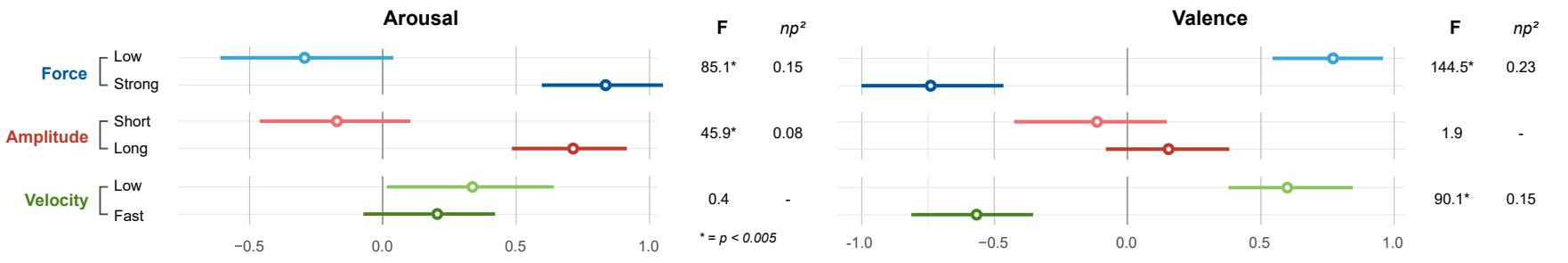

Fig. 4. Effect sizes with $95 \%$ confidence intervals of individual ratings for valence and arousal on Study 1 . The $x$-axis shows the mean effect of each characteristic on arousal and valence, with their bootstrap confidence intervals.

\subsubsection{Arousal/Valence ratings}

We analyze the results in two steps by conducting 1) a twoway repeated-measure MANOVA with Valence and Arousal as combined dependent variables and 2) $2 \times 2 \times 2$ repeatedmeasure ANOVA, on the Valence and on the Arousal data to determine effects on individual measures (effect sizes are shown as $\eta_{p}^{2}$ ). The confidence intervals of standard deviations per trial and per participant on the arousal ( $c i=$ $0.31)$ and on the valence $(c i=0.34)$ indicate that individual's ratings of the same stimulus are consistent.

The MANOVA indicates significant main effects on the individual characteristics VELOCITY $\left(F_{(2,32)}=45.12\right.$, $\left.\mathrm{p}<0.001 ; W_{i l k s}^{\prime} \Lambda=0.91, \eta_{p}^{2}=0.09\right)$, FORCE $\left(F_{(2,32)}=120.1\right.$, $\left.\mathrm{p}<0.001 ; w^{\prime} \Lambda=0.67, \eta_{p}^{2}=0.32\right)$, AMPLitude $\left(F_{(2,32)}=24.2\right.$, $\left.\mathrm{p}<0.001 ; w^{\prime} \Lambda=0.91, \eta_{p}^{2}=0.09\right)$. The MANOVA reveals significant interaction effects for VELOCITY $\times$ FORCE $\left(F_{(2,32)}=\right.$ 6.23, $\mathrm{p}<0.001 ;$ Wilks $\left.^{\prime} \Lambda=0.98, \eta_{p}^{2}=0.02\right)$ and AMPLITUDE X FORCE $\left(F_{(2,32)}=3.0, \mathrm{p}<0.001 ; w^{\prime} \Lambda=0.98, \eta_{p}^{2}=0.01\right)$.

Valence: Individual ANOVAs find a significant main effect of Velocity on Valence $\left(F_{(1,8)}=90.1, p<0.001, \eta_{p}^{2}=\right.$ $0.15)$, with the $16 \mathrm{~cm} / \mathrm{s}$ parameter resulting in a lower average valence $(\mu=-0.6, \sigma=0.8)$ than the $3.8 \mathrm{~cm} / \mathrm{s}$ parameter $(0.6,1.0)$. FORCE also has a significant effect $\left(F_{(1,8)}=\right.$ $\left.144.5, p<0.001, \eta_{p}^{2}=0.23\right)$. A low Force results in a higher valence $(0.8,1.3)$ than a strong Force $(-0.7,1.3)$. The effect of AMPLITUDE on the valence is not significant, with close results between long $(0.12,1.2)$ and short amplitude $(0.0,1.0)$. An interaction between AMPLITUDE $\mathrm{X}$ FORCE is revealed $\left(F_{(1,8)}=5.6, p<0.001, \eta_{p}^{2}=0.01\right)$.

Arousal: There is a significant effect of AMPLITUDE on arousal $\left(F_{(1,8)}=45.9, p<0.001, \eta_{p}^{2}=0.08\right)$. A short Amplitude results in a lower arousal $(\mu=-0.2, \sigma=1.4)$ than a long Amplitude $(0.7,13)$. There is a significant effect on FORCE $\left(F_{(1,8)}=85.1, p<0.001, \eta_{p}^{2}=0.15\right)$, with an arousal stronger with a high Force $(0.8,1.3)$ than low Force $(-0.3,1.3)$. No effect is found for VELOCITY, with an arousal similar for low and high velocity $(0.3,1.4)$. Finally, ANOVA reveals an interaction effect on arousal of the combined factors VELOCITY $X$ FORCE $\left(F_{(1,8)}=11.2, p<0.001, \eta_{p}^{2}=0.02\right)$.

In Figure 4, we report effect sizes of dynamic movements with $95 \%$ confidence intervals of individual ratings for both valence and arousal. The $\mathrm{x}$-axis shows the mean effect of each factor on arousal and valence. Intervals indicate all plausible values, their midpoint being about seven times more likely than their endpoints [73].

\subsubsection{Individual Differences}

Individual differences in touch perception may have an impact on the perception of the emotions [70], [74]. ANOVA suggests that the arousal/valence rating is not impacted for participants who declare liking to be touched during social communication $(\mu=3.6, \sigma=1.8)(1$ :Don't like at all, 7:Like a lot) or who like to communicate through touch $(\mu=3.4$, $\sigma=1.7)$. ANOVA does not reveal an effect of gender on arousal $\left(F_{(1,253)}=1.5\right)$, but it reveals an effect of Gender on valence $\left(F_{(1,253)}=9.4, p<0.001, \eta_{p}^{2}=0.01\right)$, where Female participants rated a higher valence $(\mu=0.3, \sigma=1.4)$ than male participants $(\mu=0.2, \sigma=1.4)$. Participants were also asked to give confidence ratings for "Globally, how difficult was it to perceive emotions?" (1: Not difficult, 7: Very difficult), which they found globally difficult ( $\mu=5.0, \sigma=1.5)$.

\subsection{Discussion}

This study investigates the impact of generated touch on the perception of arousal and valence in a context-free situation.

From Figure 3-b, we observe that low FORCE is mainly associated to emotion with low arousal while a strong FORCE is linked to emotions with high arousal and negative valence. Similarly, AMPLITUDE mainly influences arousal while touch VELOCITY tends to mainly influence valence ratings. Our results (Figure 4) further suggest that increasing the FORCE augments the arousal and reduces the valence, increasing the AMPLITUDE seems to mainly increase arousal, and increasing the VELOCITY seems to reduce the valence.

Our results match the pilot studies. First, positive emotions (high valence) are conveyed through softer touch (low FORCE, slow Velocity) [28]. Then, negative emotions involve strong touch (high FORCE) [9]. Finally, high positive or negative excitement is communicated through strong dynamic touch (high FORCE, high VELOCITY, high AMPLiTUDE) [7].

None of our generated touches convey emotions corresponding to the bottom-left quadrant, which is inline with previous studies between humans, as well as with technology-oriented studies [37], [70]. Clearly, the emotions with low arousal/valence are characterized by physiological deactivation [75] and are generally less conveyed through touch in human-human communication (e.g., we don't communicate our sadness through touch, but may use touch to express our empathy to a friend feeling sad). These emotions are usually communicated through other non-verbal communication cues, such as facial expression [25].

We also observe that three stimuli $(V+A-F+, V-A-$ $F+, V+A+F-)$ are close to the center of the circumplex model and can be considered as conveying "neutral" emotion.The other stimuli cover three of the four quadrants. In particular, the perception of the generated touch $V-A+F$ - is in the TopRight quadrant and can be associated with an emotion such as Happiness. The perception of the generated touch $V-A-F-$ 
is in the Bottom-Right quadrant and can be associated with emotions such as Calm or Boredom. Finally, the perception of three generated touches $(V+A+F+, V-A+F+, V+A-F+)$ are in the Top-left quadrant which is linked to emotions with high arousal and low valence such as Anger.

On Figure 3-b we circle the points for the Simple TYPE stimuli that were obtained during the pilot study. We can observe that the stimuli stay within the same quadrant as in the pilot study. Stimuli that were perceived previously as "neutral", that are located in the $-1 / 1$ range on arousal and valence, are still perceived as such.

From the analysis of the position of the emotional rating in the circumplex model, four distinct areas in the circumplex model emerge. They roughly correspond to the emotion labels Anger, Happiness and Calm as well as Neutral. Given our results, the smallest set of generated touches to convey distinct emotions are respectively $V+A+F+, V-A+F-$ , $V-A-F-$ and $V-A-F+$. We thus keep these four generated touches for further analysis in the next experiment.

\section{Study 2: InVestigating Generated TOUCHES WITH CONTEXT CUES}

We now explore how context cues and generated touches influence the perception of valence and of arousal. Embedding context in a perceptive study is difficult as it introduces many factors that can create bias. To circumscribe the information provided by the contextual cues, we follow a scenario-based induction technique [76]. This technique allows specifying non-ambiguous contexts. It is often used to conduct perceptive studies [25] to gather multimodal corpora [76]. Each context is simple enough to correspond to one emotion. It may not correspond to ecological settings, but since it corresponds to one value, this makes it possible to manipulate one factor at a time.

We use the four representative generated touches found in Study 1. From their position in the circumplex, they can be associated to four basic distinct emotions: Anger $(V+A+F+)$, Happiness $(V-A+F-)$, Calm $(V-A-F-)$ and Neutral $(V-A-F+)$.

Each context was associated to one emotion through a textual scenario and a facial expression (associated to the emotion) of a virtual agent (Figure 5). Using facial expressions of emotions allows us to study the emotion congruence between facial and touch stimuli [13], [60]. We considered 8 scenarios adapted from previous studies. [5], [76]: two different textual scenarios illustrate each of these emotions. We provide examples of scenarios in the appendix.

We also selected one facial expression for each of these emotions [18], which is displayed on a female 3D character. The choice of using a female agent was motivated by previous works in human-to-human touch communication suggesting disparities in cross-gender touch perception [77], [78] and was also motivated by users' agent preferences in ECA literature [79]. We validated the facial expressions through a survey with 16 participants. We presented static images of the facial expressions and for each of them asked two questions on a 7-point Likert scale to assess Arousal/Valence perception: Is the emotion conveyed by this avatar pleasant? (1 - Not pleasant at all, 7 - Very pleasant) and Is the emotion conveyed by this avatar intense? (1 - Not intense at all, 7 - Very intense). This survey confirms that each facial expression lies within a different quadrant of the arousal/valence space and corresponds to the expected emotion. The Angry facial emotion lies in the top-left quadrant, the Happy in the top-right, Calm in the bottom-right and Neutral in the center.

\subsection{Experimental Design}

The device and experimental apparatus are similar to the one described in the previous studies, but the protocol differs slightly to introduce the context cues. First, the textual scenario is shown on the screen. Then, the tactile stimulus is presented at the same time as the facial expression of the virtual agent. The facial expression is a static image displayed on the same screen of the textual scenario, on its side; Its display lasts the time of the touch. In total, there are 16 conditions. Each combination of the four stimuli (generated touches), TOUCH STIMULI with four context pairs (Congruent scenario + facial expression) CONTEXT are presented in Latin-square order to the participants. Finally, 16 right-handed volunteers $(6 \mathrm{~F})$, participated in the study, with a mean age of $28(\sigma=3.7)$. We told the participants that the touch was coming from the virtual agent present on the screen.

\subsection{Results}

Figure 3-c illustrates the position of the perception of the stimuli on the circumplex. Labels on the point indicate the CONTEXT (scenario and facial expression) while the colors are associated to TOUCH STIMULI .

\subsubsection{Arousal/Valence Ratings}

The confidence interval of standard deviations per stimulus on the arousal $(c i=0.21)$ and on the valence $(c i=0.16)$ dimensions are relatively low. To determine effects on individual measures, we analyze the results with the same methodology as in Study 1.

The MANOVA suggests significant main effects on all the factors CONTEXT $\left(F_{(2,32)}=4.48, \mathrm{p}<0.001 ; W_{i l k s}{ }^{\prime} \Lambda=\right.$ $\left.0.90, \eta_{p}^{2}=0.53\right)$ and Touch STIMUli $\left(F_{(2,32)}=17.1, \mathrm{p}<0.001\right.$; $\left.w^{\prime} \Lambda=0.67, \eta_{p}^{2}=0.18\right)$. The MANOVA does not reveal significant interaction effect of the combination of TOUCH STIMULi X CONTEXT .

Valence: Individual ANOVAs show a significant main effect of CONTEXT on valence $\left(F_{(1,4)}=9.9, p<0.001, \eta_{p}^{2}=\right.$ 0.09), with the Anger context resulting in lower average

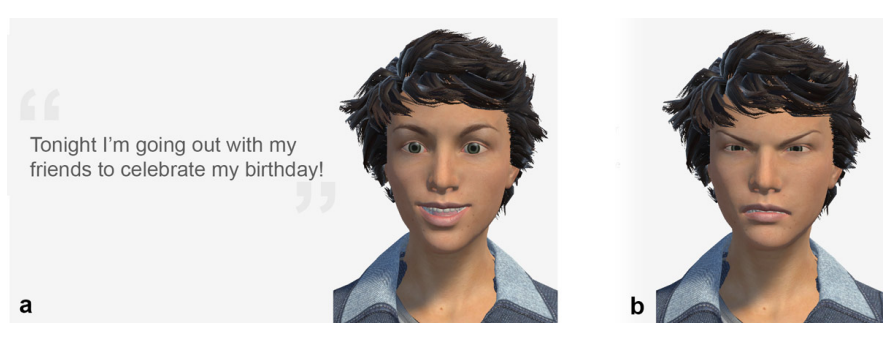

Fig. 5. Context cues as presented to the user. a) Text scenario on the left side and facial expression on the right (here Happy) b) Example of an Angry facial expression 
valence $(\mu=-0.3, \sigma=1.2)$, and Calm and Neutral the highest valence $(0.6,1.3)$. TOUCH STIMULI also have an effect on valence $\left(F_{(1,4)}=11.7, p<0.001, \eta_{p}^{2}=0.21\right)$, with a higher valence for the touch stimulus $V-A+F$ - (Happy) $(0.9,1.3)$ and a lowest one for touch stimulus $V+A+F+$ (Anger) $(-0.6,1.2)$.

Arousal: An interaction is found between CONTEXT and Touch Stimuli $\left(F_{(1,4)}=3.1, p<0.001, \eta_{p}^{2}=0.06\right)$. The mean valence varies from $\mu=-0.5$ (Participant $10 \sigma=1.2$ ) to $\mu=1.3$ (Participant 7, $\sigma=1.1$ ). ANOVA does not reveal a significant effect of CONTEXT on arousal $(\mu=0.3, \sigma=1.6)$. TOUCH STIMULI have a significant effect on arousal $\left(F_{(1,4)}=8.1, p<\right.$ $\left.0.001, \eta_{p}^{2}=0.17\right)$, with the $V+A+F+$ (Anger) touch stimulus having the highest arousal $(1.1,1.4)$ and the $V-A-F-(C a l m)$ stimulus the lowest $(-0.6,1.4)$.

\subsubsection{Individual Differences}

Similarly to our previous studies, ANOVA suggests that the individual differences such as Gender or the participant's response on whether they like to be touched during social communication $(\mu=3.5, \sigma=1.2)$ do not impact the arousal/valence ratings. Participants find the task of detecting emotions equally difficult $(\mu=5.1, \sigma=1.5)$ as in Study 1 . We find individual differences, when rating the touch stimuli, with some participants perceiving touch as more pleasant or more intense.

\subsubsection{Position on the Circumplex Model}

We can notice that the positions for each generated touch are consistent with the results obtained in Study 1. They remain in the same quadrant as when perceived in a contextfree setting. Stimuli with $V-A+F$ - touch sit within the 'high arousal, high valence' quadrant (top-right). Stimuli with $V+A+F+$ touch sit within the 'high arousal, low valence' quadrant (top-left). Stimuli with $V-A-F$ - touch are in the 'low arousal, high valence' quadrant. The stimuli with $V+A+F$ touch are located around 0 arousal and within the $-1 / 1$ range of valence.

The perception of a given generated touch remains in the same quadrant of the arousal/valence space regardless of the context cues, nevertheless the context cues modulate the perception of touch, even for non congruent (touch, context) pairs. For instance, the Calm CONTEXT lowers the perception of the generated touch corresponding to Anger $(V+A+F+)$ along the arousal dimension, while increasing the perception along the valence dimension. This result is also present for the three other generated touches. The results in the top-right quadrant (high arousal/high valence) show some confusion in the perceived emotions when the context cues and touch stimuli are not congruent. When the TOUCH STIMULi $V-A+F+$ is performed, the Calm context has a higher arousal/valence than the Happy context cue.

\subsection{Discussion}

Our results suggest that touch has a higher impact on the perception of emotion in terms of valence and arousal than context cues, but that context modulates touch perception. This finding is inline with the literature indicating that when non-congruent cues (e.g "Anger" touch with calm context cue) are presented, participants do not seem to merge stimuli but rather consider them independently and select one cue as the dominant one [80]. This choice depends on a variety of factors such as individual differences or the task being performed [80].

Our results show that there is some confusion between high valence/high arousal touch and contextual cues, e.g., between Calm and Happiness. This is in line with the literature. The perception of non-verbal positive emotional cues often shows a higher level of confusion than for negative emotions [81]. Facial expressions of positive emotions often share the same signals (smile, raised eyebrows, crow's feet, etc.) and several studies suggest that smile dynamics might be more important to discern those expressions [25]. However in our experiment, the facial expression of the agent was static, presented as an image. The lack of dynamism in the facial expression may have created confusion in the perception of the stimuli.

Other studies that present tactile stimulus with facial expressions of an ECA suggest that participants seem to rely on the visual modality for the evaluation of valence and on the kinesthetic modality for the arousal one [19], [82]. Our results do not reproduce this finding. This might be explained by the fact that our tactile stimuli are produced differently than those used in the previous studies (moving the participant's arm in [82] or inflating an object in a participant's hand in [19]). The touch generated by the robotic arm used in our study combine amplitude, velocity, and contact force against the participant's arm. It is closer to human touch than in the touch used in these studies.

\section{CONCLUSION AND FUtURE WORK}

We now discuss the results and the limitations of our studies considering the choice of the characteristics, parameters and context cues. We also propose directions for future work.

\subsection{Relevance of the design choices}

This paper investigates machine-to-human touch movements. The main difficulty is the gap between the enormous size of the space of touch characteristics of the human movement in comparison with the limited capabilities of existing haptic devices. It was unclear how existing actuated and haptic devices could communicate emotions through touch. Our approach consisted of reducing the scope of the study to render the problem tractable with the risk of not capturing important elements to convey different degrees of arousal and valence through touch. Based on the human-tohuman literature and the analysis of haptic technologies, we selected a robotic arm augmented with an artificial hand to study the impact of four touch characteristics AMPLITUDE, VELOCITY, FORCE and TYPE on the perception of arousal and valence. Even though these characteristics are somehow limited, they are enough to convey persistent arousal and valence combinations. For sake of simplicity, we have only used two values for each characteristic. Future work should investigate different values of these characteristics.

An important finding of our studies is that this device is relevant to convey at least four consistent combinations of arousal and valence through touch by only manipulating the values of these three characteristics. Moreover, our results share similarities with those found in human-to-human 
touch studies [9]. For instance, a "hit" with a strong force is perceived as conveying Anger [7]; touch with strong force is perceived as less pleasant and more intense than touch with light force. This is an interesting result because it demonstrates that using robotic technologies to simulate rich touch events is a promising direction.

While we were concerned with the social acceptance of the robotic devices due to a potentially negative attitude toward robots [83], some participants reported that it was more acceptable for them to be touched by a robotic arm than by a human $(\mu=5.81, \sigma=1.06)$. For instance, one participant indicated: "I know that such a device can't have mean intentions, hence I prefer to be touched by it rather than by a human". In other words, the device is not only efficient in communicating emotions through touch but appears socially acceptable, at least in the context of our study. This aspect should be further investigated in a study to measure the intentions participants attribute to touch when interacting with a virtual agent or a robot.

\subsection{Future directions}

Our device has some limitations and we considered a small number of touch characteristics and parameters to reduce the scope of our studies. This opens several directions for future work. For instance, we limited the amplitude of a generated touch to ensure touching only the forearm of the participants and to remain in the limits of the robotic arm amplitude. Similarly, the maximum velocity corresponds to the limit of the robotic arm speed. The range of the force values could be increased, but within the limit of not hurting users. Future work is necessary to conclude on the uppermost limit for each characteristic, taking into account that sharing the workspace of a large robotic device is subject to safety norms (ISO 15066) that cannot be ignored.

Other kinds of actuated devices can be used to generate touches on the user. For instance, we prototyped a miniaturized device called Mobilimb [84] (Figure 6, right). Mobilimb is a robotic actuator shaped as a finger. It can be plugged onto a mobile phone and can touch the user on the wrist or the back of the hand with various amplitudes, velocities and forces. Due to miniaturization, the parameter ranges are smaller than when using a robotic arm (Mobilimb enables a maximum Force of about $0.3 \mathrm{~N}$, an Amplitude of $15 \mathrm{~cm}$ on the skin (with a precision of $\pm 5 \mathrm{~mm}$ ) and a Velocity of $4 \mathrm{~cm} / \mathrm{s}$ ). Mobilimb can be used to reproduce an emotional touch with a portable device and demonstrates the technical feasibility of our approach. A user study remains to be carried out for testing the emotional perception of the generated touches. Nevertheless, these devices explore a new way for devices to perform affective touch to humans, and might improve affective grounding between a robot and a human [85].

Future work could test the perception of arousal and of valence of other characteristics such as the temperature, the texture, the gestures, etc. For instance, "surface in contact" could have an impact on the perception of valence as physical interactions, such as a hug, involve a long mutual contact on larger body surface areas. The location of a touch movement on the body can also influence the perceived arousal and valence. Other types of gestures could also be considered, such as "Grabbing". Social communication

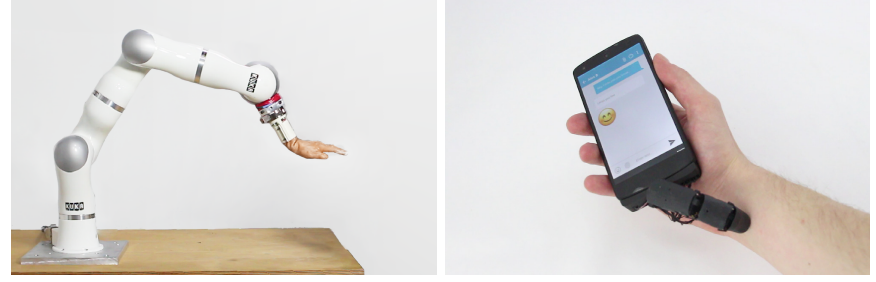

Fig. 6. Left. KUKA LWR4+ robotic arm used in our studies. Right. Mobilimb, prototye of miniature device for touch.

also involves gestures such as tapping someone's back to convey affect without being too intimate. Future research could also investigate how adding other characteristics such as temperature and texture would influence the perception of arousal and of valence.

In Study 2, we presented the expression of the virtual agent and the touch as the superposition of two stimuli. To give the impression the agent produced required a complex setup. For instance, seeing the agent performing the touch on the user requires synchronicity and congruence between full scale virtual animation and the movement of the robot. Future work should explore the notion of agency of the agent and to measure if the participants had the impression the touch came from the agent.

Complementary work is needed to consider additional dimensions regarding the participants such as their culture. Although the meaning of touch varies depending on one's culture [86], its impact on emotional touch perception is not yet clear enough. Some results suggest that emotions conveyed by touch might be similar between cultures [7], [8], but others [87] suggest the opposite.

\subsection{Using Generated Touches for Interaction}

We can ask ourselves which touch characteristics and parameters would maximize the range of perceived emotions during human-machine interaction. But the study of a given touch gesture should require considering its combination with the parameters of all other characteristics, thus dramatically increasing the number of stimuli to be tested. For example, we found that while FORCE changes drastically the perception of touch, this perception depends on combinations with other parameters. A low FORCE conveys higher valence emotions with low arousal and a strong FORCE suggests high arousal and negative valence emotions. However, a combination of a high FORCE with high AMPLITUDE and low VELOCITY conveys a high valence emotion (see Figure 3-b).

Moreover, we can observe variability between participants on their perception of the valence and of the arousal of a given touch stimulus. Some users only use a small region of the circumplex model, while others use the whole area. While participants may not perceive the same arousal/valence for a given stimulus, they still perceive a similar change in emotion perception (e.g., a more aroused emotion) when one touch parameter is modified (e.g., a stimulus with faster velocity). So the results across participants are better explained by looking at the differential in the perception of emotions. This is a common result for emotion perception from multimodal cues [42]. The variability between individuals has to be considered to propose a 
credible experience and could be resolved by performing an initial calibration phase where users rate example stimuli.

In our study 2 we used both textual scenarios and facial expressions of a virtual agent. However, other factors might impact the perception of emotions during human-agent interaction such as acoustic cues, body parameters, or the task that is being performed. Moreover, users' perception of the context vary with regards of their current emotional state, their perception of the ECA with whom they are communicating, or the global experience of the interaction (e.g. is it a stressful environment). These factors have to be taken into consideration when designing touch interactions, in particular, to decide which touch should be performed and how it can potentially be perceived.

\section{References}

[1] P. Ekman, "Strong evidence for universals in facial expressions: a reply to russell's mistaken critique." 1994.

[2] K. R. Scherer and H. Ellgring, "Are facial expressions of emotion produced by categorical affect programs or dynamically driven by appraisal?" Emotion, vol. 7, no. 1, pp. 113-130, 2007.

[3] R. Banse and K. R. Scherer, "Acoustic profiles in vocal emotion expression." Journ. of personality and social psychology, vol. 70, 1996.

[4] T. Vogt, E. André, and N. Bee, "Emovoice-a framework for online recognition of emotions from voice," in Int. Tutorial and Research Workshop on Perception and Interactive Technologies for Speech-Based Systems. Springer, 2008, pp. 188-199.

[5] N. Dael, M. Mortillaro, and K. R. Scherer, "Emotion expression in body action and posture." Emotion, vol. 12, no. 5, 2012.

[6] N. Bianchi-Berthouze, "Understanding the role of body movement in player engagement," Human-Computer Interaction, vol. 28, 2013.

[7] M. J. Hertenstein, D. Keltner, B. App, B. a. Bulleit, and A. R. Jaskolka, "Touch communicates distinct emotions." Emotion (Washington, D.C.), vol. 6, no. 3, 2006.

[8] M. J. Hertenstein, R. Holmes, M. McCullough, and D. Keltner, "The communication of emotion via touch." Emotion, vol. 9, 2009.

[9] G. K. Essick, F. McGlone, C. Dancer, D. Fabricant, Y. Ragin, N. Phillips, T. Jones, and S. Guest, "Quantitative assessment of pleasant touch," Neuroscience and Biobehavioral Reviews, vol. 34, no. 2, pp. 192-203, 2010.

[10] L. S. I Morrison, Löken and H. Olausson, "The skin as a social organ," Experimental brain research, vol. 204, no. 3, 2010.

[11] J. B. Van Erp and A. Toet, "Social touch in human-computer interaction," Frontiers in digital humanities, vol. 2, p. 2, 2015.

[12] A. H. Basori, A. Bade, M. S. Sunar, D. Daman, and N. Saari, "Haptic vibration for emotional expression of avatar to enhance the realism of virtual reality," in 2009 Int. Conf. on Computer Technology and Development, vol. 2. IEEE, 2009.

[13] G. Huisman, "Social touch technology: A survey of haptic technology for social touch," IEEE Transactions on Haptics, 2017.

[14] R. M. Baños, C. Botella, M. Alcañiz, V. Liaño, B. Guerrero, and B. Rey, "Immersion and emotion: their impact on the sense of presence," Cyberpsychology \& behavior, vol. 7, no. 6, 2004.

[15] J. Broekens, M. Heerink, H. Rosendal et al., "Assistive social robots in elderly care: a review," Gerontechnology, vol. 8, no. 2, 2009.

[16] E.-L. Sallnäs, "Haptic feedback increases perceived social presence," in Int. Conf. on Human Haptic Sensing and Touch Enabled Computer Applications. Springer, 2010, pp. 178-185.

[17] I. Ahmed, V. Harjunen, G. Jacucci, E. Hoggan, N. Ravaja, and M. M. Spapé, "Reach out and touch me: Effects of four distinct haptic technologies on affective touch in virtual reality," in 18th ACM Int. Conf. on Multimodal Interaction. ACM, 2016.

[18] R. Niewiadomski, S. J. Hyniewska, and C. Pelachaud, "Computational models of expressive behaviors for a virtual agent," Social emotions in nature and artifact. Oxford University Press, 2013.

[19] T. W. Bickmore, R. Fernando, L. Ring, and D. Schulman, "Empathic touch by relational agents," IEEE Transactions on Affective Computing, vol. 1, no. 1, pp. 60-71, 2010.

[20] A. Haans and W. A. IJsselsteijn, "The virtual midas touch: Helping behavior after a mediated social touch," IEEE Transactions on Haptics, vol. 2, no. 3, pp. 136-140, 2009.
[21] K. R. Thórisson, "Communicative humanoids: a computational model of psychosocial dialogue skills," Ph.D. dissertation, Massachusetts Institute of Technology, 1996.

[22] G. Cannata, M. Maggiali, G. Metta, and G. Sandini, "An embedded artificial skin for humanoid robots," in Multisensor Fusion and Integration for Intelligent Systems, 2008. MFI 2008. IEEE Int. Conf. on. IEEE, 2008, pp. 434-438.

[23] T. L. Chen, C.-h. King, A. L. Thomaz, and C. C. Kemp, “Touched by a robot: An investigation of subjective responses to robot-initiated touch BT - 6th ACM/IEEE Int. Conf. on Human-Robot Interaction, HRI 2011, March 6, 2011," 2011.

[24] L. S. Löken, J. Wessberg, F. McGlone, and H. Olausson, "Coding of pleasant touch by unmyelinated afferents in humans," Nature neuroscience, vol. 12, no. 5, pp. 547-548, 2009.

[25] M. Ochs, C. Pelachaud, and G. Mckeown, "A user perceptionbased approach to create smiling embodied conversational agents," ACM Trans. Interact. Intell. Syst., vol. 7, no. 1, Jan. 2017.

[26] J. A. Russell, "Evidence of convergent validity on the dimensions of affect." Journal of personality and social psychology, vol. 36, 1978.

[27] A. H. Crusco and C. G. Wetzel, "The midas touch: The effects of interpersonal touch on restaurant tipping," Personality and Social Psychology Bulletin, vol. 10, no. 4, pp. 512-517, 1984.

[28] G. K. Essick, A. James, and F. P. McGlone, "Psychophysical assessment of the affective components of non-painful touch," Neuroreport, vol. 10, no. 10, pp. 2083-2087, 1999.

[29] T. Nguyen, R. Heslin, and M. L. Nguyen, "The meanings of touch: Sex differences," Journal of Communication, vol. 25, no. 3, 1975.

[30] B. App, D. N. McIntosh, C. L. Reed, and M. J. Hertenstein, "Nonverbal channel use in communication of emotion: how may depend on why." Emotion, vol. 11, no. 3, p. 603, 2011.

[31] J. B. Walther and J. K. Burgoon, "Relational communication in computer-mediated interaction," Human communication research, vol. 19 , no. $1,1992$.

[32] C. E. Connor, S. S. Hsiao, J. Phillips, and K. Johnson, "Tactile roughness: neural codes that account for psychophysical magnitude estimates," Journal of Neuroscience, vol. 10, 1990.

[33] G. Huisman, A. D. Frederiks, and D. Heylen, "Affective touch at a distance," in Affective Computing and Intelligent Interaction (ACII), 2013 Humaine Association Conf. on. IEEE, 2013, pp. 701-702.

[34] K. E. MacLean, "Designing with haptic feedback," in ICRA'00, IEEE Int. Conf. on Robotics and Automation, vol. 1. IEEE, 2000.

[35] C. Cascio, F. McGlone, S. Folger, V. Tannan, G. Baranek, K. A. Pelphrey, and G. Essick, "Tactile perception in adults with autism: a multidimensional psychophysical study," Journal of autism and developmental disorders, vol. 38, no. 1, pp. 127-137, 2008.

[36] K. Salminen, V. Surakka, J. Raisamo, J. Lylykangas, R. Raisamo, K. Mäkelä, and T. Ahmaniemi, "Cold or hot? how thermal stimuli are related to human emotional system?" in Int. Workshop on Haptic and Audio Interaction Design. Springer, 2013, pp. 20-29.

[37] G. Wilson, D. Dobrev, and S. A. Brewster, "Hot Under the Collar: Mapping Thermal Feedback to Dimensional Models of Emotion," Proc. of the 2016 Conf. on Human Factors in Computing Systems, 2016.

[38] Y. Yoo, T. Yoo, J. Kong, and S. Choi, "Emotional responses of tactile icons: Effects of amplitude, frequency, duration, and envelope," in World Haptics Conf. (WHC), 2015 IEEE. IEEE, 2015.

[39] J. Cha, M. Eid, A. Barghout, A. Rahman, and A. El Saddik, "Hugme: synchronous haptic teleconferencing," in Proc. of the 17th ACM international conference on Multimedia, 2009.

[40] P. Lemmens, F. Crompvoets, D. Brokken, J. Van Den Eerenbeemd, and G.-J. de Vries, "A body-conforming tactile jacket to enrich movie viewing," in EuroHaptics Conf., 2009 and Symposium on Haptic Interfaces for Virtual Environment and Teleoperator Systems. World Haptics 2009. Third Joint. IEEE, 2009, pp. 7-12.

[41] J. B. Van Erp and H. Van Veen, "A multi-purpose tactile vest for astronauts in the international space station," in Proc. of eurohaptics. Dublin, Ireland: ACM, Press, 2003, pp. 405-408.

[42] G. Wilson and S. A. Brewster, "Multi-moji: Combining thermal, vibrotactile \& visual stimuli to expand the affective range of feedback," in Proc. of the 2017 CHI Conf. on Human Factors in Computing Systems. ACM, 2017, pp. 1743-1755.

[43] J. Tewell, J. Bird, and G. R. Buchanan, "The heat is on: A temperature display for conveying affective feedback," in Proc. of the 2017 CHI Conf. on Human Factors in Computing Systems, ser. CHI '17. ACM, 2017.

[44] K. Hyunjung and L. Woohun, "Shade pixel: Interactive skin for ambient information displays," CHI'09, pp. 1-4, 2009. 
[45] Y. Jansen, "Mudpad: fluid haptics for multitouch surfaces," in CHI'10 Extended Abstracts on Human Factors in Computing Systems. ACM, 2010, pp. 4351-4356.

[46] C. Vaucelle, L. Bonanni, and H. Ishii, "Design of haptic interfaces for therapy," in Proc. of the SIGCHI Conf. on Human Factors in Computing Systems, ser. CHI '09. ACM, 2009.

[47] J. N. Bailenson, N. Yee, S. Brave, D. Merget, and D. Koslow, "Virtual interpersonal touch: expressing and recognizing emotions through haptic devices," Human-Computer Interaction, vol. 22, no. 3, pp. 325-353, 2007.

[48] F. Gosselin, C. Andriot, J. Savall, and J. Martín, "Large workspace haptic devices for human-scale interaction: A survey," in Int. Conf. on Human Haptic Sensing and Touch Enabled Computer Applications. Springer, 2008.

[49] Y. Yokokohji, R. L. Hollis, and T. Kanade, "Wysiwyf display: A visual/haptic interface to virtual environment," Presence, vol. 8, no. 4, pp. 412-434, 1999.

[50] E. Vonach, C. Gatterer, and H. Kaufmann, "Vrrobot: Robot actuated props in an infinite virtual environment," in Virtual Reality (VR), 2017 IEEE. IEEE, 2017, pp. 74-83.

[51] B. Araujo, R. Jota, V. Perumal, J. X. Yao, K. Singh, and D. Wigdor, "Snake charmer: Physically enabling virtual objects," in Proc. of the TEI'16: Tenth Int. Conf. on Tangible, Embedded, and Embodied Interaction. ACM, 2016, pp. 218-226.

[52] S. Šabanović, C. C. Bennett, W.-L. Chang, and L. Huber, "Paro robot affects diverse interaction modalities in group sensory therapy for older adults with dementia," in 2013 IEEE 13th International Conference on Rehabilitation Robotics (ICORR), pp. 1-6.

[53] S. Yohanan, M. Chan, J. Hopkins, H. Sun, and K. MacLean, "Hapticat: exploration of affective touch," in Proc. of the 7th international conference on Multimodal interfaces. ACM, 2005, pp. 222-229.

[54] T. Shibata and K. Tanie, "Physical and affective interaction between human and mental commit robot," in Robotics and Automation, 2001. Proc. 2001 ICRA. IEEE Int. Conf. on, vol. 3. IEEE, 2001.

[55] H. Sumioka, A. Nakae, R. Kanai, and H. Ishiguro, "Huggable communication medium decreases cortisol levels," Scientific reports, vol. 3, 2013.

[56] C. J. Willemse, G. Huisman, M. M. Jung, J. B. van Erp, and D. K. Heylen, "Observing touch from video: The influence of social cues on pleasantness perceptions," in Int. Conf. on Human Haptic Sensing and Touch Enabled Computer Applications. Springer, 2016.

[57] T. Hirano, M. Shiomi, T. Iio, M. Kimoto, I. Tanev, K. Shimohara, and N. Hagita, "How do communication cues change impressions of human-robot touch interaction?" Int. Journal of Social Robotics, vol. 10, no. 1, pp. 21-31, 2018.

[58] L. H. Kim and S. Follmer, "Swarmhaptics: Haptic display with swarm robots," in Proc. of the 2019 CHI Conf. on Human Factors in Computing Systems. ACM, 2019, p. 688.

[59] N. Ravaja, V. Harjunen, I. Ahmed, G. Jacucci, and M. Spapé, “Feeling touched: Emotional modulation of somatosensory potentials to interpersonal touch," Scientific reports, vol. 7, 2017.

[60] G. Huisman, M. Bruijnes, J. Kolkmeier, M. Jung, A. D. Frederiks, and Y. Rybarczyk, "Touching virtual agents: embodiment and mind," in Int. Summer Workshop on Multimodal Interfaces. Springer, 2013, pp. 114-138.

[61] N. Nguyen, I. Wachsmuth, and S. Kopp, “Touch perception and emotional appraisal for a virtual agent," in Proc. Workshop Emotion and Computing-Current Research and Future Impact, 2007.

[62] A. Mehrabian, "Pleasure-arousal-dominance: A general framework for describing and measuring individual differences in temperament," Current Psychology: Developmental, Learning, Personality, Social, vol. 14, 1996.

[63] L. Kertay and S. L. Reviere, "Touch in context," Touch in psychotherapy: Theory, research, and practice, pp. 16-35, 1998.

[64] T. Bänziger, M. Mortillaro, and K. R. Scherer, "Introducing the geneva multimodal expression corpus for experimental research on emotion perception," Emotion, vol. 12, no. 5, p. 1161-79, 2012.

[65] N. Fourati and C. Pelachaud, "Perception of emotions and body movement in the emilya database," IEEE Transactions on Affective Computing, vol. 9, no. 1, pp. 90-101, 2018.

[66] C. Krahe, M. Drabek, Y. Paloyelis, and A. Fotopoulou, "Affective touch and attachment style modulate pain: A laser-evoked potentials study," Philosophical Transactions of the Royal Society, 2016.

[67] C. J. Willemse, A. Toet, and J. B. van Erp, "Affective and behavioral responses to robot-initiated social touch: toward understanding the opportunities and limitations of physical contact in humanrobot interaction," Frontiers in ICT, 2017.
[68] I. ISO, "Iso/ts 15066 robots and robotic devices-collaborative robots," Int. Organization for Standardization, 2016.

[69] S. E. Jones and A. E. Yarbrough, "A naturalistic study of the meanings of touch," Communications Monographs, vol. 52, 1985.

[70] G. Wilson and S. A. Brewster, "Multi-moji: Combining thermal, vibrotactile \&\#38; visual stimuli to expand the affective range of feedback," in Proc. of the 2017 CHI Conf. on Human Factors in Computing Systems, ser. CHI '17. ACM, 2017, pp. 1743-1755.

[71] C. Caroni and P. Prescott, "Sequential application of wilks's multivariate outlier test," Applied Statistics, pp. 355-364, 1992.

[72] J. A. Russell, "Measures of emotion," in The measurement of emotions. Elsevier, 1989

[73] G. Cumming, Understanding the new statistics: Effect sizes, confidence intervals, and meta-analysis. Routledge, 2013.

[74] H. Seifi and K. E. Maclean, "A first look at individuals' affective ratings of vibrations," in World Haptics Conf. IEEE, 2013.

[75] R. Reisenzein, "Pleasure-arousal theory and the intensity of emotions." Journal of Personality and Social Psychology, vol. 67, 1994.

[76] T. Bänziger and K. R. Scherer, "Introducing the geneva multimodal emotion portrayal (gemep) corpus," Blueprint for affective computing: A sourcebook, 2010.

[77] D. S. Stier and J. A. Hall, "Gender differences in touch: An empirical and theoretical review." Journal of personality and social psychology, vol. 47, no. 2, p. 440, 1984.

[78] B. Major and R. Heslin, "Perceptions of cross-sex and same-sex nonreciprocal touch: It is better to give than to receive," Journal of Nonverbal Behavior, vol. 6, no. 3, pp. 148-162, 1982.

[79] J. Forlizzi, J. Zimmerman, V. Mancuso, and S. Kwak, "How interface agents affect interaction between humans and computers," in Proceedings of the 2007 conference on Designing pleasurable products and interfaces, 2007, pp. 209-221.

[80] B. E. Stein and M. A. Meredith, The merging of the senses. The MIT Press, 1993.

[81] D. A. Sauter, N. M. McDonald, D. N. Gangi, D. S. Messinger et al., "Nonverbal expressions of positive emotions," Handbook of positive emotions, pp. 179-198, 2014.

[82] Y. Gaffary, J.-C. Martin, and M. Ammi, "Perception of congruent facial and kinesthetic expressions of emotions," in 2015 Int. Conf. on Affective Computing and Intelligent Interaction (ACII). IEEE, 2015.

[83] T. Nomura, T. Kanda, T. Suzuki, and K. Kato, "Psychology in human-robot communication: An attempt through investigation of negative attitudes and anxiety toward robots," in 13th IEEE Int. Workshop on Robot and Human Interactive Communication, ROMAN 2004.

[84] M. Teyssier, G. Bailly, C. Pelachaud, and E. Lecolinet, "Mobilimb: Augmenting mobile devices with a robotic limb," in 31st ACM Symposium on User Interface Software and Technology. ACM, 2018.

[85] M. F. Jung, "Affective grounding in human-robot interaction," in Proc. of the 2017 ACM/IEEE Int. Conf. on Human-Robot Interaction.

[86] J. T. Suvilehto, E. Glerean, R. I. Dunbar, R. Hari, and L. Nummenmaa, "Topography of social touching depends on emotional bonds between humans," Proc. of the National Academy of Sciences, vol. 112, no. 45, pp. 13811-13 816, 2015.

[87] A. Cranny-Francis, "Semefulness: a social semiotics of touch," Social Semiotics, vol. 21, no. 4, pp. 463-481, 2011.

Marc Teyssier is currently a Post-doctorant research at Saarland University. He did his Ph.D at Télécom Paris, Institut polytechnique de Paris. His research explore how affective communication can be mediated through anthropomorphic devices.

Gilles Bailly is a CNRS researcher at Sorbonne Université. His research is at the crossroad of Human-Computer Interaction, Robotics, Skill acquisition and Decision making. He designs novel interaction techniques and build predictive models of performance with a focus on the transition from novice to expert behavior.

Catherine Pelachaud is a Research Director at ISIR-CNRS laboratory at Sorbonne Université. Her research interests include embodied conversational agent, nonverbal communication (face, gaze, and gesture), expressive behaviors, and socio-emotional agents.

Eric Lecolinet is a Professor at the LTCl laboratory of Télécom Paris - Institut Polytechnique de Paris. His research interests lie in HumanComputer Interaction, particularly on interaction techniques for small and large devices, gestural interaction and tactile feedback. 